Official Publication of the International Association for Cognitive Education and Psychology

www.ia-cep.org

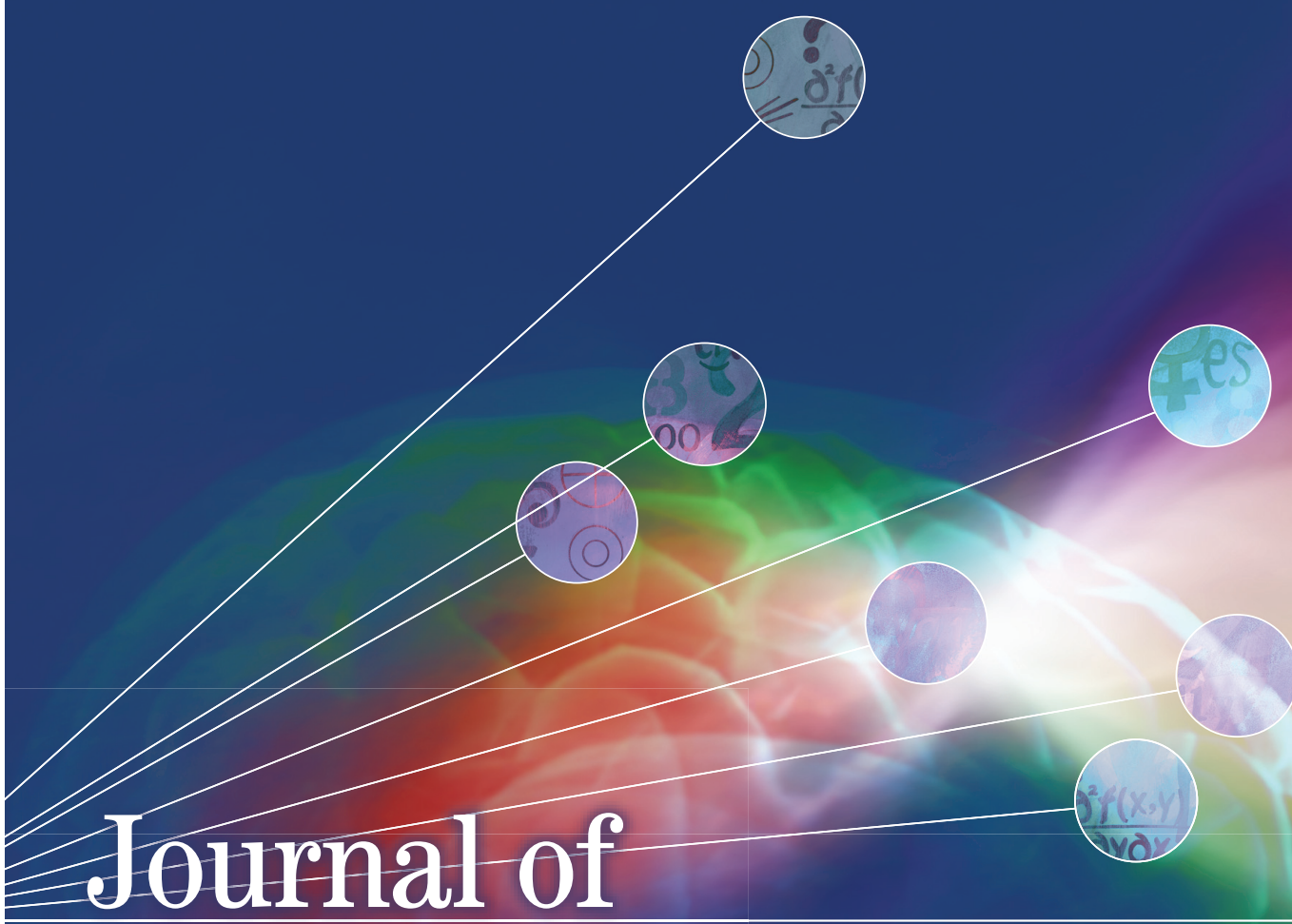

\title{
Cognitive Education and Psychology
}

With the Compliments of Springer Publishing Company, LLC 


\title{
Theory and Research
}

\section{Children's Inductive Reasoning: Developmental and Educational Perspectives}

\author{
Patrick Perret \\ Aix Marseille Université, Aix en Provence, France
}

\begin{abstract}
Omnipresent in human thought, inductive reasoning consists in (a) detecting regularities, (b) abstracting relations, and (c) deriving general rules. In the first part of this article, I attempt to identify the basic mechanisms underpinning inductive reasoning and the reasons why it is so central to the workings of intelligence. I then go on to describe several factors that researchers in developmental psychology believe may contribute to the development of inductive reasoning. Each factor's influence is illustrated by its potential contribution to the resolution of Raven's Progressives Matrices. In the third and final part, I examine the issue from an educational perspective, showing how developmental hypotheses can inform different types of interventions designed to foster inductive reasoning in children.
\end{abstract}

Keywords: reasoning; induction; cognitive development; cognitive education; Raven's Progressive Matrices

$\mathrm{I}$ nductive reasoning is central to the functioning of children's intelligence and constitutes a driving force of cognitive development. It is one of the main levers by which children understand how the world around them works through the abstraction of its underlying rules. Theories of induction integrate arguments from various approaches and disciplines such as philosophy, history of sciences, mathematical modeling, artificial intelligence, and psychology (Feeney \& Heit, 2007). The aim of the present review is to identify the mechanisms that may govern the development of inductive reasoning in children. It is also to show that a finer grained knowledge of these developmental factors can illuminate our 
interpretation of children's difficulties on inductive reasoning tasks and guide educational strategies to support the growth of inductive skills.

\section{WHAT IS INDUCTIVE REASONING?}

"Reasoning is, in part, effortful perception" (Sternberg, 1986, p. 287).

The concept of inductive reasoning (IR) refers to the cognitive activity of generating inferences that classically meet two criteria: (a) direction (IR moves from the observation of particular cases to the formulation of more general rules) and (b) level of confidence (IR is a form of reasoning under uncertainty because it involves forming hypotheses about rules). As such, it is frequently defined in opposition to deductive reasoning: Deductive inferences move from the general to the particular and are logical necessities. In deductive arguments, the premises provide absolute grounds for accepting the conclusion. Borrowed from Galotti, Komatsu, and Voelz (1997), the syllogisms provided in Figure 1 neatly illustrate this double contrast.

The deductive or inductive nature of a problem can therefore be broadly identified based on these two criteria (but see Heit, 2007, for extensive discussion of potential pitfalls in the delimitation of problems' status). From a psychological point of view, several areas of research converge toward the assumption that distinct cognitive processes underpin the production of these two kinds of inferences.

The psychometric approach draws on individual differences of performance on batteries of tests to identify latent sources of variance reflecting separate (although interconnected) aptitudes. This line of research has consistently shown that inductive and deductive reasoning tests fell into distinct registers: To properly fit the data from individual differences, theoretical models of cognitive abilities have to incorporate this distinction (Carroll, 1993). On the experimental front, Heit and Rotello (2010) have found that inductive and deductive inferences are differentially affected by experimental manipulations such as varying the similarity between categories used in the premise and the conclusion. With reference to the two-process accounts of reasoning (Evans, 2008), the authors suggested that inductive and deductive judgments both recruit heuristic and analytic processes but in different proportions (deduction relying more heavily on slower analytic processes). Finally, neuropsychological data also argue in favor of a dissociative view. Goel and Dolan (2004) studied the respective neuroanatomy of inductive versus deductive reasoning. Both were found to involve left prefrontal cortex, but deduction was associated with an increased activation of Broca's area, whereas induction mainly recruited left dorsolateral prefrontal cortex.
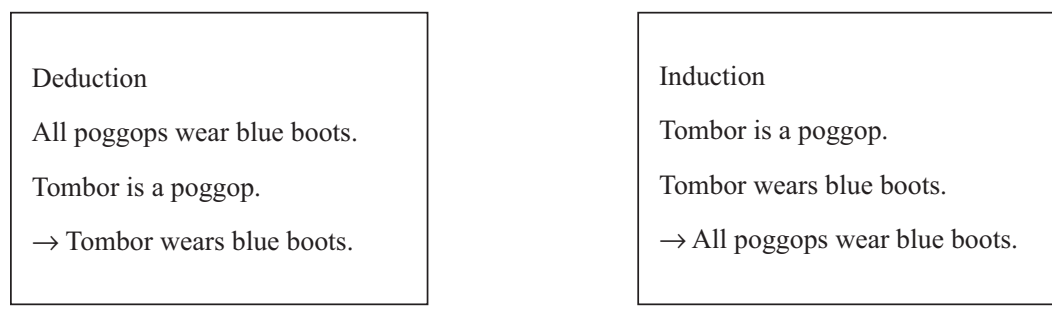

FIGURE 1. Examples of deductive and inductive inferences. 
Inductive inferences play a part in a wide range of daily cognitive activities. They include the following:

- Feature attribution based on categorical generalization: when we hypothesize that all the members of a given category share a property after observing that one or several of them do

- Analogical reasoning: when we hypothesize that a known relation between two concepts can tell us about the relation linking two other concepts

- Causal reasoning: when we hypothesize that two events are linked by a causal relationship based on the observation of their conditional occurrence

- Probabilistic judgment: when we modulate our predictions about future events after taking a sample of similar events into account

The sheer diversity of cognitive contexts involving the generation of inductive inferences suggests that they represent one of the commonest forms of human thought. Moshman $(1995,2004)$, however, stresses that a distinction should be made between the mere production of inferences—often an implicit and largely unconscious activity—and actual reasoning, when we subject our inferences to a test of their validity: "Reasoning is epistemically selfconstrained thinking" (Moshman, 2004, p. 223). Therefore, reasoning is conceived of not as an automatic process but as an activity that is costly in attentional resources and where intellectual effort is required to justify inferences. Kuhn (2013) goes one step further, asserting that it is not only a conscious activity but one that is explicitly goal oriented, whether this goal is simply understanding a situation or taking a decision that implies action.

\section{INDUCTIVE REASONING AND THE DEVELOPMENT OF INTELLIGENCE}

"Inductive reasoning enables one to detect regularities, rules, or generalizations and, conversely, to detect irregularities. This is one way in which we structure our world" (Klauer, Willmes, \& Phye, 2002, p. 1).

The development of both fluid intelligence (the ability to resolve novel problems in working memory [WM]) and crystallized intelligence (the ability to elaborate and mobilize knowledge in long-term memory) depends on IR. A long tradition of psychometric research has shown that of all the dimensions of intellectual functioning, fluid intelligence is the one with the highest general factor loading. IR is, in turn, the factor with the highest fluid intelligence loading (Carroll, 1993). This means that a considerable proportion of individual variation in intellectual efficiency is linked to variability in IR ability, which explains the enduring popularity of Raven's Progressive Matrices (RPM) as a tool for assessing intellectual functioning (Oakland \& Hu, 1992). Raven based his reasoning test on Spearman's (1927) conception that the eduction of relations (i.e., the ability to discern a relationship between two stimuli) constitutes the core dimension of the general intelligence factor and determines the ability to make meaning out of confusion. IR is therefore classically assessed using tests that require relations to be abstracted. In both matrix tests and series completion tests (e.g., logical series), children have to figure out the laws governing the ordering of a set of items. The componential theory of inductive reasoning, proposed by Sternberg and Gardner (1983), articulates the psychometric and information-processing research traditions. Within this framework, latent 
variables are thought to emerge from factor analyses because of commonalities in information processing requirements of tasks usually used to assess inductive competence. The analyses, conducted with analogy completion tasks, classification tasks, and series completion tasks, have led the authors to propose a model of IR based on five underlying processes: encoding (of the attributes characterizing elements), inference (of relations between elements), mapping of relations (seeking out a higher order general rule), application (of this rule from a new element to an hypothetical ideal completion), and response comparison (of this hypothetical completion with response options). Research conducted by Mareschal and colleagues (Leech, Mareschal, \& Cooper, 2007; Thomas \& Mareschal, 2001) further suggested that relations are conceived of in the mind as transformations between states of the world: Because a relation is not a perceptual datum, it has to be mentally represented as the transformation of one entity into another.

IR lies at the heart of fluid intelligence because we can only resolve a problem once we have understood the relational system on which it is based. When solving complex and unfamiliar problems for which no specific content knowledge is applicable (i.e., the hallmark of fluid intelligence), inductive processes play a major role: It is by generating hypothetical rules, based on observations, that relational systems can be abstracted. Molnár, Greiff, and Csapó (2013) provided empirical support for this view. They studied developmental trends in problem-solving abilities and found that the correlations with IR efficiency were stable over time.

IR not only enables the online resolution of new problems but also the inducement of more general schemas promoting the transfer of resolution strategies between problems of the same class (Phye, 1990). As such, it also contributes to the development of crystallized intelligence. The seminal work of Holyoak on analogy as a powerful learning mechanism neatly illustrates this point (Gentner \& Holyoak, 1997; Holyoak \& Thagard, 1997): Detecting underlying similarities between seemingly different situations favors the acquisition of higher order knowledge. For Holland, Holyoak, Nisbett, and Thagard (1986), the mental models that result from inductive processes are schematic representations of phenomena-be they physical, social, numerical, or logical — that the mind needs to understand to adapt to them. These cognitive representations may be only selective approximations but nonetheless allow the mind to engage in a form of adaptive anticipation: "The key feature of mental models is that they include structures and processes that can simulate the transitions that occur in phenomena being modeled" (p. 327).

In the light of this conception, the theories inductively developed by scientists to understand and represent the real world can be seen as an advanced form of mental model, with explicit and formalized construction mechanisms. However, scientists are not the only people to devise theories about the world around them. Gopnik and Wellman's (1994) theorytheory states that the knowledge elaborated by children resembles scientific theory in both its structure (e.g., its quest for an explanation, its requirement of coherence, its capacity for prediction and interpretation) and the inductive processes that lead to its elaboration, such as the analysis of covariation patterns between events or the effects of intervention (Cook, Goodman, \& Schulz, 2011). In the course of development, these naive theories are fuelled not only by children's autonomous IR but also by conceptual input from their cultural communities. Neo-Vygostkian approaches of theoretical learning in classrooms try to articulate these two sources of knowledge, by affording pupils opportunities to use IR to cope with problem situations, while at the same time instilling discipline-related concepts (Karpov, 2013). 


\section{HOW DOES INDUCTIVE REASONING DEVELOP?}

We now know that children start implicitly producing inductive inferences at a very early age, as Schulz, Goodman, Tenenbaum, and Jenkins (2008) have demonstrated. In the initial phase of their experiment, these authors showed preschoolers colored building blocks that sometimes emitted a sound when they came into contact with each other, instantiating specific, but arbitrary, causal relations (e.g., when a blue block came into contact with a red one, it made a train noise, and when a yellow block came into contact with a blue one, it produced the sound of a siren). In the second phase, the children were introduced to a novel block that contradicted the rules that had hitherto governed the outcome of different block contact combinations. As early as 4 years, children recognized when abstract rules had been violated and sought a coherent causal explanation.

These early inferences seem to be modulated by the same variables as they are in adults. For instance, one of the known triggers of inductive generalization is the number of observations: The larger our sample of observations, the more likely we are to risk inferring the existence of an underlying general rule. Lawson and Fisher (2009) demonstrated that in favorable conditions, sample size can be seen to have an effect on inference production as early as 5 years. More generally, their results (Lawson \& Fisher, 2011) suggest that children's inductive inferences, like those of adults, are extremely sensitive to the regularity of the observed phenomena. However, from a purely logical stance, our inductive inferences can be systematically questioned: Even when grounded on multiple and consistent observations, the available evidence could always support alternative interpretations based on new concepts and justify alternative projections. The fact that some inferences appear more "natural" than others despite their logical equivalence is a long-lasting theoretical issue (known as the new riddle of induction) for both philosophers (Goodman, 1955) and psychologists (Sternberg, 1983).

Although the basic inductive mechanisms appear to emerge at a fairly young age, IR becomes noticeably more efficient with age. When Csapó (Csapó, 1997; Molnár et al., 2013) administered a battery of inductive tests (number and verbal series, verbal and number analogies, exclusion, etc.) to children in a series of cross-sectional studies, he observed a substantial improvement, leading him to conclude that "inductive reasoning develops during a broad age range and that the developmental process covers the whole period of elementary and secondary education" (Csapó, 1997, p. 623). Furthermore, correlations between performances on the different tests suggested that comparable processes were implemented to solve the problems. Composite IR scores derived from these tests were predictive both of knowledge acquired a year later (thus confirming the contribution of IR to the development of crystallized intelligence) and of progress in problem solving (thus confirming the contribution of IR to the development of fluid intelligence).

These data establishing the extent and impact of IR development raise the question of exactly how this development occurs. In the following sections, I therefore list the various possible contributing factors. I illustrate the contribution of each one to IR development in terms of its impact on performances on the RPM test.

\section{The Relational Shift}

As we saw earlier, IR is closely associated with the ability of the human mind to grasp relations and systems of relations. According to Gentner (1988), children's cognitive development is characterized by a radical shift in this area. Although their attention is initially drawn to 
perceptual similarities between objects (e.g., conceiving that a ball is red in the same way that an apple is red), they later graduate from appearance similarity to relational similarity (e.g., conceiving that a ball is on the table in the same way that a book is on the desk). This relational shift sets the path for the development of analogical reasoning: Analogy entails finding a structural alignment in which the key similarities lie in relations between elements, more than in the features of objects. However, Gentner's hypothesis has been widely discussed in the wake of a set of studies indicating that children as young as 3 or 4 years can take account of relational analogies, providing that the conditions in which the problems are presented favor their detection (see Goswami, 2010, for a summary). Rattermann and Gentner (1998) then posited that the perceptual-to-relational shift should not be viewed as a transition between two universal, age-defined developmental stages but as a sequence that is reiterated in each separate knowledge domain. According to these authors, children are only able to go beyond their initial focus on object properties to take relational similarities on board once they have acquired a degree of expertise and mastered the necessary concepts. More recently, Bulloch and Opfer (2009) have suggested that developmental improvements in resolving inductive reasoning problems reflect not just greater ease in considering relations per se but also, and above all, greater flexibility and sensitivity to the validity of surface versus structural cues, depending on which form of similarity is most relevant in the given situation.

RPM success, for instance, depends on being able to alternate between appearance similarities in some items and relational similarities in others. The hierarchy of matrix difficulty is itself compatible with the perceptual-to-relational shift hypothesis in that the simplest items (i.e., those that can be successfully resolved by young children) require participants to complete perceptual patterns or recognize the constancy of different shape attributes, whereas the most complex items (i.e., those that can only be successfully resolved by older children) rely on relational analogies (e.g., rules of distribution or quantitative progression).

\section{The Development of Relational Processing Ability}

The theory of relational complexity developed by Halford and colleagues (Halford, Wilson, \& Phillips, 1998, 2010) implies that the ability to process relations in WM is another major contributing factor to progress in IR. When a child has to resolve a problem that requires fluid intelligence, the relational schemas needed to grasp the problem structure are elaborated in WM.

According to Halford's theory, it is processing capacity of WM that determines the degree of relational complexity the mind is able to represent. This complexity can be defined by the number of variables that interact within a given relational system and must therefore be processed in parallel within the same mental model. By looking at the number of variables that have to be represented simultaneously, we can therefore determine the level of relational complexity. Halford's theory states that fourth-order relations represent the highest level of complexity the human mind can cope with. Halford, Baker, McCredden, and Bain (2005) explored the limits of human processing capacity by asking adult participants to interpret statistical interactions featuring increasing numbers of variables. Participants had to complete the final sentence of a verbal description with the term that most appropriately described the data presented in a graphical representation of the interaction. Results showed that when the problem required more than four variables to be processed simultaneously, participants' performances ceased to be above chance level. 
In children, relational complexity theory predicts a gradual increase in processing capacity along the following developmental timeline: The ability to process relations involving a single variable is thought to emerge at around 1 year, the processing of binary relations around 2 years, and that of ternary relations around 5 years, whereas the median age for the ability to process quaternary relations is 11 years. This steady rise in the number of variables that children can handle within a single representation is thought to increase the scope and complexity of the concepts they can master. It is also believed to extend the reasoning they can bring to bear by enabling them to base their inferences on increasingly complex mental models that mirror the relational systems at work in the problems they have to solve.

One of the main sources of variation in RPM difficulty is precisely the number of rules that have to be combined to grasp the overall structure and construct a representation of the missing shape (Carpenter, Just, \& Shell, 1990). In support of this hypothesis, Dauvier, Bailleux, and Perret (2014) recently demonstrated that the effect of age on RPM performances is partly mediated by improvements in relational processing capacity in WM. Furthermore, the task these authors developed to assess relational integration capacity in children (Relational Integration Level Assessment Task [RILAT]) proved to be more predictive of RPM performance than classic WM tasks (such as the Backward Digit-Span Task), which primarily assess storage capacity (Perret, Dauvier, Bailleux, \& Thomachot, 2013).

\section{Strategic Development}

Owing to our limited WM capacity, our ability to deal with complex problems partly depends on the strategies we adopt to sidestep that complexity. For instance, segmentation strategies (Halford et al., 1998) consist in breaking the task down into several steps, none of which exceeds the individual's processing capacity. This means that the relational system no longer has to be grasped in its entirety but can be understood piecemeal, in several successive, manageable segments.

So central is this strategic dimension to our cognitive functioning that it lies at the core of recent models of development. For Siegler (1996, 2000), learning and development processes are subtended by overlapping waves of strategy use through the discovery of new strategies, more frequent use of the most effective strategies, or increasingly efficient strategy implementation. In Siegler's model, development is not conceived of as a series of abrupt changes in the way that children grasp a problem situation but rather as gradual increases or decreases in the probability of mobilizing strategies that can coexist in a child's mind. Furthermore, microgenetic studies have revealed that these gradual changes sometimes take place implicitly, with children adopting a new approach to a problem situation without being fully aware they are using a new strategy (e.g., Siegler \& Stern, 1998).

As far as IR is concerned, Whitebread (1996) showed that improved performances on a discrimination-learning task, where children had to identify a rule by analyzing its invariants, were associated with a gradual transformation in the strategies participants used to test their hypotheses. Research on the factors that contribute to effective RPM resolution has also helped to shed light on this strategic dimension. Snow (1980) identified a constructive matching strategy that consists in mentally preparing an ideal response and then comparing it with the responses that are actually available and an elimination strategy that consists in comparing the features of the matrix elements with those of the possible responses, thus arriving at a default response through a process of elimination. By analyzing response times coupled 
with eye movements, Vigneau, Caissie, and Bors (2006) were able to confirm that individual variations in RPM efficiency are closely linked to these strategic factors: Participants with the best performances spent proportionately more time analyzing the matrix than they did analyzing the response options and strategically varied the amount of time spent on each item as a function of its perceived complexity.

\section{The Development of Inhibitory Control}

The age-related improvement in the inhibitory control of thoughts is another general factor that is often mentioned. Here, the term inhibition refers to the active process of suppressing interfering representations in WM. Given the structural limitations of WM capacity, it is vital to keep a tight control of resources, only allocating them to the most relevant informations. Inhibition is therefore given a key role in several general models of cognitive development (e.g., Bjorklund \& Harnishfeger, 1990), for in problem-solving situations, the representations elaborated in WM are often guided either by salient information in the perceptual environment or else by familiar strategies triggered by the context. Many Piagetian tasks, for instance, set traps for children, who therefore have not only to apply logical-mathematical knowledge but also to inhibit the processing of the problem's misleading dimensions (Houdé, 2000). Improved performances may therefore reflect not just the enhancement of the logical skills elicited by these tasks but also greater inhibitory control of thoughts (Dempster, 1991).

The contribution of inhibitory control to IR development has mainly been explored in studies of analogical reasoning. Morrison, Doumas, and Richland (2011) used a neurally inspired computer model to show that an improvement in the inhibition level of the model's WM system could help to explain the progress in children's ability to resist distraction from perceptual similarities in favor of relation-based reasoning. In an extension of this hypothesis, Thibaut, French, and Vezneva (2010) reported that between the ages of 6 and 14 years, children performing an analogical reasoning test become increasingly successful in resisting the perceptual distractors introduced into the response options to compete with relational matches. In other words, the age-related increase in IR effectiveness may rest on the parallel development of the ability to abstract relations and the ability to inhibit the processing of surface information that often hinders this abstraction. Furthermore, at the other end of the lifespan, results of the study by Viskontas, Morrison, Holyoak, Hummel, and Knowlton (2004) suggest that the decline in IR associated with cognitive aging may be the consequence of a comparable interaction between inhibitory functions and relational processing.

RPM resolution is also subject to interference of this sort. Some response options are more distracting than others, accounting for the most frequent errors reported in the test manual. According to Jarosz and Wiley (2012), the correlation that is regularly observed between RPM and WM test performances could be linked to the fact that both types of test rely on the ability to manage attentional resources and resist interference. By experimentally manipulating the degree of interference for each item (i.e., adding or subtracting the most frequently selected incorrect responses), the authors showed that the presence of salient distractors has the greatest impact on participants with the poorest WM performances. An additional analysis of eye movements revealed that the participants with the best WM performances were not only better able to resist the highly distracting incorrect responses but were also more likely to adopt a constructive matching strategy. 


\section{Metalogical Development}

Metalogical development refers to improvements in children's epistemological understanding that their knowledge (and that of others) can be generated by different types of cognitive activity (e.g., perception, remembering, forgetting, reasoning, strategy use). The empirical studies conducted by Pillow (e.g., Pillow \& Pearson, 2012) reveal a marked age-related improvement in this conception of the mind as an active system of knowledge construction. If we take the example of the distinction between guesswork, deduction, and inductive inference, although children can acknowledge that deduction is superior to guesswork as early as 5-6 years, the explicit comprehension of inductive processes is more complex and is achieved far later, mainly because children first have to recognize that knowledge can be uncertain.

As we saw earlier, Moshman's $(1995,2004)$ model assumes that improvements in children's reasoning affect not only the complexity of their inferences but also their level of metacognitive awareness. In turn, this growing awareness affords children more rigorous control over the inferences they make. Moshman claims that social interactions can encourage the self-reflective activity that supports metalogical development because they force children to justify their inferences and accept the notion that different trains of thought can lead their interlocutors to different conclusions. Age-related improvements in IR may therefore be partly subtended by an increasingly acute awareness of the inferential processes that are brought into play, which at the same time promotes their regulation and generalization.

During RPM resolution, this awareness may result in an increasingly explicit identification of the inferences used to resolve the matrices. When Carpenter et al. (1990) analyzed the RPM matrices, they discovered that despite their apparent diversity, the transformations undergone by the figures were governed by just five very general rules: constant in a row, quantitative pairwise progression, figure addition or subtraction, distribution of two values, and distribution of three values. We can assume that the better participants become at identifying these metarules, the easier they find it to apply specific rules to specific matrices. In line with this hypothesis, by experimentally manipulating the order in which the items were presented, Verguts and De Boeck (2002) were able to show that participants clearly do better when they have already resolved items using a comparable rule. More recently, Wiley, Jarosz, Cushen, and Colflesh (2011) found that WM capacity is most closely correlated with individual differences when items require the discovery of a new combination of rules. The identification of a metarule appears to makes its transposition to subsequent items both more probable and less cognitively costly. Meanwhile, adopting a dynamic assessment perspective, Carlson and Wiedl $(1979,1992)$ observed an improvement in RPM performances when conditions favored inferential awareness (i.e., by asking the children to verbalize their reasoning - thinking aloud—during the task and justify their inference after choosing one of the responses).

\section{Changes in Thinking Dispositions}

Dispositions are relatively stable psychological tendencies that allow us to differentiate between the functioning of different individuals. Insofar as we are allowed choices in our daily lives, these personal inclinations guide those choices in a relatively coherent manner. The notion of disposition (or trait) is a familiar one to psychologists investigating socioemotional functioning or personality development. Research by Perkins and colleagues (Perkins, 1995; Perkins, Tishman, Ritchart, Donis, \& Andrade, 2000) has shown that this concept can also 
help us understand variations in the effectiveness of intellectual functioning. His central argument is that implementing intelligent behavior requires not only intellectual aptitude but also the ability to detect occasions for thinking (i.e., sensitivity) and the motivation to make and control rigorous inferences (i.e., inclination). Variations in the ability or willingness to explore a problem situation in depth, test hypotheses, adopt a strategic attitude, take on board several perspectives, or tolerate uncertainty may not only help to explain individual differences but also drive age-related improvements in reasoning.

Reasoning is an optional process that is extremely costly in terms of cognitive effort. This means that individuals only fully engage in it if they are acutely aware of its expected benefits and have developed appropriate epistemological values. Kokis, Macpherson, Toplak, West, and Stanovich (2002) looked at whether children aged 10-11 years and 13 years engaged in heuristic (intuitive) or in analytic processing to resolve problems involving inductive, deductive, and probabilistic reasoning. Results highlighted an age-related increase in analytic responding. The study's independent variables included measures of cognitive ability and thinking dispositions. Consistent with Perkins' proposals, results of the regression analyses showed that thinking dispositions explained a specific proportion of variance in analytic responding after controlling for variance shared with cognitive ability.

RPM resolution is obviously sensitive to thinking dispositions. Implementing a constructive matching strategy involves processing systems of relations that increase in complexity across the items, forcing participants to mobilize considerable WM resources. In the test manual, Raven, Raven, and Court (1998) highlight this dispositional dimension, claiming that one of the main sources of error is the reluctance to devote mental energy to resolving abstract problems. Vodegel-Matzen, van der Molen, and Dudink (1994) found that highscoring individuals typically spent more time on resolving the RPM items than low scorers. In a similar vein, Kagan, Pearson, and Welch (1966) had previously highlighted the influence of cognitive style (impulsive vs. reflective) on IR test performances.

\section{FOSTERING THE DEVELOPMENT OF INDUCTIVE REASONING}

"We can debate the exact nature of the process, but few would refute the claim that it is through application and practice that reasoning skills improve" (Kuhn, Katz, \& Dean, 2004, p. 197).

Because they apply across a wide range of contexts, inductive processes are prime targets for cognitive education interventions designed to foster the development of children's adaptive resources. Not only is IR a lever for constructing subject knowledge (e.g., through the discovery of the rules that govern language structure or number relations) but the ever increasing complexity and diversification of information sources in today's world means that it is also vital to possess rigorous inductive processes if-to paraphrase Raven et al. (1998) - we are to "extract meaning from chaos." For instance, children's ability to master the new technological devices that now play such an important part in their lives relies mainly on their ability to use induction to fathom out the systems of arbitrary rules on which they are based.

Several intervention studies have empirically demonstrated that it is possible to enhance the efficiency of IR. The studies conducted by Nisbett and colleagues (Nisbett, Fong, Lehman, $\&$ Cheng, 1987, for a review) provided evidence that (a) reasoning skills of college students can be trained and that (b) training effects can transfer to a wide range of problem contents. 
Those results, which contradicted the view of human cognition as inherently domain specific, also set the path for a reasonably optimistic perspective regarding the cognitive education of reasoning (Nisbett, 2009). The work of de Koning, Hamers, Sijtsma, and Vermeer (2002) further indicates that it is possible to educate IR processes in school-age children, through specially designed activities. In the following sections, I give examples of three such methods, showing how they promote the main factors for progress identified in developmental psychology research.

\section{Fostering the Relational Shift: The Example of the Transformation Box}

As we have seen, IR is based on the processing of relations. Presumably, therefore, it can only be implemented by individuals who can look beyond surface similarities and focus instead on relational similarities. This attentional switch encourages children to form representations of general rules or A:B:C:D-type analogies. According to Paour (1995), "the abstraction of arbitrary relations is the most common and most familiar manifestation of intelligence at work" (p. 267). He therefore designed a device intended to promote the abstraction of relations: the Transformation Box (Paour, 1992).

This is essentially a large box with four holes in the top. Children can insert objects or pictures of objects into these holes, which are said to "transform" objects. Of course, no actual magic is involved: At the back of the box, the adult substitutes a new object for the one the child has introduced, but because of the way the box is designed, the substitution looks like a transformation. Each hole corresponds to a hatch from which the substituted object can be retrieved (Figure 2). The transformations are systematic, in that each hole is associated with a general rule that the adult has to follow (e.g., changing the object's color, shape, or size;

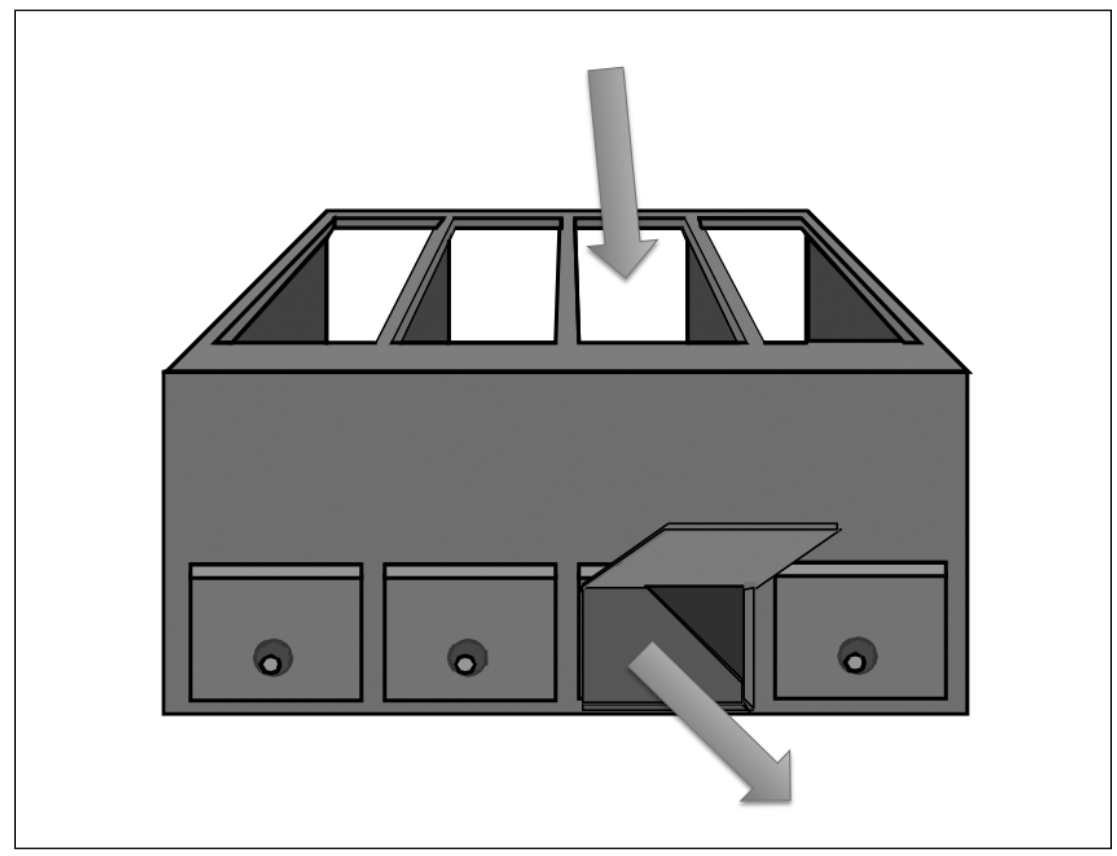

FIGURE 2. Illustration showing how the Transformation Box works. 
modifying its orientation or texture, in either an absolute or a relative way). Children first have to work out "how each hole works" through experience. They then have to anticipate the outcome of hypothetical transformations based on the rules they have managed to abstract ("Given the rule for this hole, what will the result be if we insert a particular object?"). In real life, relations are not only general but also omnidirectional, in that they can work in different ways (Halford et al., 1998), so we can also reverse the anticipation ("Given what I know about how this hole works, what object do I have to insert to obtain a particular result?”). The third and final phase consists in getting the children to coordinate a series of transformations to move from one state to another. This is a genuine problem-solving situation, in that the children have to make planned use of the systems of relations they discovered during the exploratory phase to achieve a goal.

By encouraging children to focus their attention on relational invariants from which they can abstract a general rule, the Transformation Box directly operationalizes the promotion of the relational shift. It also elegantly embodies Leech et al.'s (2007) postulate that the mind mainly conceives of relations as transformational systems. Up to now, research on the effects of training with the Transformation Box has focused on groups of children with intellectual disability (Paour, 1992). An intervention study comparing 22 children with a mental age of 4-7 years and a control group that received the same number of individual training sessions revealed a significant effect on RPM performances in a posttest conducted 4 months after the intervention had ended (effect size not reported). These encouraging results for children with intellectual disability suggest that it might be useful to extend the study of training effects to typically developing children.

\section{Fostering Relational Integration: The Example of Relevant Playful Activities}

Play situations can potentially offer occasions for developing general intellectual skills that are just as beneficial as cognitive education methods. Hessels-Schlatter (2010) claims that play helps children to see the advantages of adopting efficient processing strategies, whereas transposing these strategies to different games helps to generalize them. Moreover, the defense mechanisms that characterize failing schoolchildren's attitudes to learning are less salient in these situations. As such, games provide an opportunity for modifying children's attributional beliefs (i.e., the way they interpret their successes and failures in terms of effort and strategy use). This can only take place, however, once the cognitive processes needed to solve them have been analyzed to select the ones that most closely match the objective being pursued.

As we saw earlier, relational integration is one of the potential factors for IR development. Relational integration refers to the ability to represent the interaction of several variables within a coordinated system. Mackey, Hill, Stone, and Bunge (2011) recently carried out an intervention study with children aged 7-10 years that focused on precisely this dimension of cognitive functioning. The children in the experimental group underwent 16 sessions, each lasting $75 \mathrm{~min}$, during which they were given the opportunity to practice 12 different games, all of which required several relations or rules to be coordinated. For example, the Set game consists in carefully examining 12 cards that can vary in four dimensions: symbol, color, number, and shading. The aim of the game is to form a set of three cards that are either totally identical to or totally different from each other on each of the four dimensions. The nature of each card that is selected therefore constrains 
the choice of the following cards and sets up an interaction between the different dimensions that have to be simultaneously taken into account. In this study, a control group underwent a comparable period of intensive training but featuring different games that required high information processing speed and rapid responses but were based on very simple rules. Speed, for instance, is a game that manipulates the same dimensions as Set, but the aim is to shed one's cards as quickly as possible by adding them to a central stack. There is just one rule: the card must have something in common with the top card in the stack.

IR was assessed with the Test of Nonverbal Intelligence (3rd edition; TONI-3) before and after the training. This matrix completion test probes analogical reasoning processes similar to those required by RPM items. Results revealed a significant improvement in the scores of the children in the relational integration training group (Cohen's $d=1.51$ ) but not in those belonging to the processing speed group.

\section{Fostering Strategic and Metalogical Regulation of Inferences: \\ The Developmental Program of Inductive Reasoning}

Aware of the centrality of IR in hierarchical models of intellectual functioning, Klauer and Phye (1994) came up with one of the most advanced programs for promoting its development in children. They defined IR as the discovery of regularities through the detection of similarities, differences, or a combination of the two with respect to either object attributes or relations between objects. This definition allowed them to clearly identify the different categories of problems in which this mode of thought is involved and thus to establish a taxonomy of inductive problems based on these two dimensions (i.e., attributes vs. relations and similarities vs. differences; Table 1).

The Developmental Program of Inductive Reasoning comprises 120 problems (20 per category) in various information formats: pictorial, geometric, verbal, and numerical. The program has a twofold objective. First, the child must identify the nature of the problem being posed and form an explicit representation of the category it belongs to (i.e., the program's metalogical component). Second, he or she must tackle each problem situation in an analytic way, systematically undertaking an exhaustive comparison of the elements (i.e., the program's strategic dimension).

Tomic and Kingma (1998) studied the effects of this program in 23 6-year-olds, who underwent 10 training sessions, each lasting $30 \mathrm{~min}$. A control group of same-age children of comparable intellectual level performed tasks from the regular school curriculum. The authors observed a significant effect of training on performances on the Colored RPM in a posttest administered 4 months after the end of training (effect size not reported). Another recent study (Molnár, 2011) explored the effect of this program (10 sessions lasting 20 min, spread over an 8-week period) in 90 children aged 6-8 years. The pre- and posttests featured six categories of problems inspired by Klauer and Phye's (1994) taxonomy. The effects were noteworthy not just for their size (Cohen's $d=1.12$ ) but also for their duration (a significant difference between the control group and the experimental group could still be observed in a follow-up study 1 year after the training had finished). Moreover, the training effect remained stable regardless of the children's original IR level established in the pretest. The greatest effects observed in the posttest were for problems involving the coordination of relational similarities and differences, that is, matrix problems comparable to RPM items. 
TABLE 1. Klauer and Phye's Taxonomy of Inductive Reasoning Problems

\begin{tabular}{ll}
\hline Cognitive Processes & Types of Problems \\
\hline Object attributes & \\
Detecting similarities & Class formation \\
Detecting differences & Identifying irregularities \\
Coordinating similarities and differences & Cross classification \\
Relations between objects & \\
Detecting similarities & Simple analogies, series completion \\
Detecting differences & Identifying disrupted series \\
Coordinating similarities and differences & Matrices \\
\hline
\end{tabular}

Note. From Cognitive Training for Children: A Developmental Program of Inductive Reasoning and Problem Solving by K. J. Klauer and G. D. Phye, 1994, Seattle, WA: Hogrefe. Copyright (1994) by Hogrefe Publishing. Adopted with permission.

Other intervention studies conducted by the program's authors (for a review, see Klauer \& Phye, 2008) have highlighted its effects directly on RPM performances, even among children with mild intellectual disability and, at the other end of the spectrum, among intellectually gifted children.

\section{IMPLICATIONS AND PERSPECTIVES}

In the first half of this article, I argued that IR is one of the decisive ingredients of human intelligence. For Hofstadter (2001), this fundamental ability to abstract relations through the detection of regularities represents "the very blue that fills the whole sky of cognition" (p. 499). I also argued that attempts to promote IR are more likely to succeed if they are based on the vectors for change identified by researchers. Studies in the field of developmental psychology are painting an increasingly well-documented picture of the factors that contribute to IR. The three training methods described earlier illustrate how knowledge of these factors can be used to design targeted programs. I selected these three examples not only because they have started to bring results but also because the authors of these programs rooted their design in explicit developmental hypotheses. Having such a limited selection means that although I was able to highlight some of the factors that can inform intervention programs (i.e., relational shift, relational integration, strategic and metalogical development), I necessarily neglected others, such as inhibition processes, the ability to maintain information in WM, and thinking dispositions. These factors also deserve to be taken into account not only by program designers but also by psychologists seeking to identify and overcome the obstacles that some children encounter.

Inhibitory control is a key executive function, regulating thought and behavior. By enabling children to suspend their most impulsive reactions, it gives them space to reflect on social or cognitive situations. Several studies have begun to look into the possibility of giving children general inhibition training and examine its impact on reasoning (Rueda, Rothbart, Saccamanno, \& Posner, 2005; Thorell, Lindqvist, Bergman Nutley, Bohlin, \& Klingberg, 2009). It is worth noting, however, that improvements in inhibitory control are themselves contingent on progress in metalogical understanding. For children to actively inhibit an inefficient strategy or interfering information in reasoning situations, they must first see a good reason 
for doing so. In experimental tasks designed to measure inhibition levels, such as the Stroop (1935) test, this metalogical dimension is neutralized because participants are explicitly told what they must not do. In ecologically valid reasoning situations, by contrast, the decision to inhibit a given strategy is up to the child and therefore presupposes prior recognition of its inappropriate nature. Inhibition training (for a review, see Diamond \& Lee, 2011) might nonetheless reinforce a general disposition for effortful control, which favors the adoption of more reflective attitudes in problem solving (Rothbart \& Rueda, 2005).

In my discussion of WM influence, I emphasized its relational integration component. There is both theoretical (Halford \& Andrews, 2006) and empirical (Oberauer, Süb, Wilhem, $\&$ Whittmann, 2008) reason to think that processing capacity exerts the greatest constraint on the development of reasoning. However, WM is a space not only for processing but also for the temporary storage of information, and most WM assessments take the form of span measures. Regular observations of close correlations between these storage measures and measures of fluid intelligence have recently prompted the marketing of WM training programs and a clear increase in intervention studies. Despite the proliferation of studies and even meta-analyses, it has so far proved difficult to identify the conditions in which WM training is most likely to lead to transfer effects in reasoning tasks (Cowan, 2014; Shipstead, Hicks, \& Engle, 2012). Although WM training holds out promising prospects, I suspect that it has been the focus of excessive interest. The hierarchical organization of the Cattell-Horn-Carroll model (Carroll, 1993) suggests that if our aim is to foster the development of reasoning and fluid intelligence, we would be better off giving children training in inferential processes themselves. To illustrate, in one recent and particularly well-controlled study (Bergman Nutley et al., 2011), the authors compared the effects of different types of training on 4-yearolds: progressively difficult training in nonverbal reasoning versus progressively difficult WM training. A control group was trained in the same tasks but remained at the lowest level of difficulty. The authors explored the effect of these types of training on a range of cognitive functions, including fluid intelligence, which was measured as a latent variable derived from several tests (including the Colored RPM). Only the group that was specifically trained in nonverbal reasoning displayed a significant improvement in the fluid intelligence latent variable on the posttest (Cohen's $d=0.63$ on Set B of the Colored RPM). Conversely, improved WM performances were only found in the group that had been trained in WM.

Thinking dispositions have been defined as sensitivity to thinking occasions and inclination to engage in controlled and justifiable inferential processes. It would be difficult to devise a training program for thinking dispositions per se. Nevertheless, unless children's thinking dispositions can be stimulated, it is pointless hoping for the transfer and generalization of cognitive education methods: "We may experience varying degrees of success in undertaking to teach students to reason well, but doing so is to no avail unless they believe it is important to do so [emphasis added]" (Kuhn, 2013, p. 760). For both Moshman (2004) and Kuhn (2013), changes in the way that children understand potential reasoning situations mainly occur in social interactions, when children have to justify their inferential approach: "When reasoning is situated in a social context, it undergoes scrutiny from multiple perspectives that acts as a catalyst for deeper processing and change" (Kuhn, 2013, p. 760). Feuerstein's pioneering work highlighted both the centrality of IR and the social origins of its refinements. The instruments that he developed (Feuerstein, Feuerstein, Falik, \& Rand, 2002; Feuerstein, Rand, Hoffman, \& Miller, 1980) clearly focused on induction as one of the key processes by which problems are solved. Moreover, the theory of structural cognitive modifiability envisaged 
social interactions, taking the form of mediated learning experiences or collaborative learning experiences, as proximal factors for the actualization of potentialities. The methods used to promote IR that I have described here (e.g., the Transformation Box or Klauer and Phye's program) do not simply expose children to problem situations. Instead, they set up opportunities for the children to interact with a mediating adult, who asks them about what they are doing, refrains their impulses, requires logical justifications, and praises the efforts they make. Thinking dispositions can be likened to a system of cognitive values, such as the desire to undertake rigorous, in-depth information processing before validating an inference. They are culturally transmitted in social experiences where adults interpret the reasons for the children's performances, gradually structuring their attributional belief system (Yeager $\&$ Dweck, 2012). To illustrate, in the area of IR, the disposition to compare could become a target for early intervention. Indeed, the comparison process is the main lever for abstracting relations (Markman \& Gentner, 2001). According to Klauer, the surest way of discovering regularities amid all the apparent chaos is to adopt a top-down strategy of systematic and analytic comparison. However, as Haywood points out in the design of his cognitive early education program (Brooks \& Haywood, 2003), inequalities between children are less to do with their ability to compare (a relatively straightforward process) than with their tendency to engage in spontaneous comparative behavior. The sensitivity and inclination to compare is the product of mediated learning experiences where this valuable cognitive attitude is presented in a positive light.

The main aim of this article was to show how knowledge of the factors for development identified by researchers can inform program design and guide adults' mediation strategies. The opposite is equally true. The scientific community is increasingly recognizing that intervention studies are a valuable method for overcoming problems in the causal interpretation of developmental correlational data (e.g., Zelazo, 2013). From this perspective, the challenge is not only to undertake double-blind randomized studies but also to delimit the cognitive processes that are elicited as clearly as possible to correctly attribute the effects that are observed. I should stress, however, that this need for parsimony applies to intervention studies conducted with a research goal in mind but not to interventions themselves. On the practical front, clinical and educational psychologists have every interest in using all the sources of improvement at their disposal (e.g., combining several training programs; getting the children to play games that require the coordination of relations; encouraging them to make comparisons whenever the occasion presents itself; praising them for adopting a systematic, strategic attitude; giving them a taste for intellectual effort) rather than placing all their hopes in a single method predicated on a single hypothetical key process. As this review has sought to show, IR development is the result of a myriad of influences interacting over time.

\section{REFERENCES}

Bergman Nutley, S., Söderqvist, S., Bryde, S., Thorell, L. B., Humphreys, K., \& Klingberg, T. (2011). Gains in fluid intelligence after non-verbal reasoning training in 4-year-old children-A controlled, randomized study. Developmental Science, 14, 591-601.

Bjorklund, D. F., \& Harnishfeger, K. K. (1990). The resources construct in cognitive development: Diverse sources of evidence and a theory of inefficient inhibition. Developmental Review, 10, 48-71.

Brooks, P. H., \& Haywood, H. C. (2003). A preschool mediational context: The Bright Start curriculum. In A. S.-H. Seng, L. K.-H. Pou, \& O.-S. Tan (Eds.), Mediated learning experience with children: Applications across contexts (pp. 98-132). Singapore: McGraw-Hill Education. 
Bulloch, M. J., \& Opfer, J. E. (2009). What makes relational reasoning smart? Revisiting the relational shift in cognitive development. Developmental Science, 12, 114-122.

Carlson, J. S., \& Wiedl, K. H. (1979). Toward a differential testing approach: Testing-the-limits employing the Raven matrices. Intelligence, 3, 323-344.

Carlson, J. S., \& Wiedl, K. H. (1992). The dynamic assessment of intelligence. In H. C. Haywood \& D. Tzuriel (Eds.), Interactive assessment (pp. 167-186). Berlin, Germany: Springer Verlag.

Carpenter, P. A., Just, M. A., \& Shell, P. (1990). What one intelligence test measures: A theoretical account of the processing in the Raven Progressive Matrices Test. Psychological Review, 97, 404-431.

Carroll, J. B. (1993). Human cognitive abilities: A survey offactor analytic studies. New York, NY: Cambridge University Press.

Cook, C., Goodman, N. D., \& Schulz, L. E. (2011). Where science starts: Spontaneous experiments in preschoolers' exploratory play. Cognition, 120, 341-349.

Cowan, N. (2014). Working memory underpins cognitive development, learning, and education. Educational Psychology Review, 26, 197-223.

Csapó, B. (1997). The development of inductive reasoning: Cross-sectional assessments in an educational context. International Journal of Behavioral Development, 20, 609-626.

Dauvier, B., Bailleux, C., \& Perret, P. (2014). The development of relational integration during childhood. Developmental Psychology, 50, 1687-1697.

de Koning, E., Hamers, J. H. M., Sijtsma, K., \& Vermeer, A. (2002). Teaching and transfer of inductive reasoning in primary education. Developmental Review, 22, 211-241.

Dempster, F. N. (1991). Inhibitory processes: A neglected dimension of intelligence. Intelligence, 15, 157-173.

Diamond, A., \& Lee, K. (2011). Interventions shown to aid executive function development in children 4 to 12 years old. Science, 333, 959-964.

Evans, J. S. (2008). Dual-processing accounts of reasoning, judgment, and social cognition. Annual Review of Psychology, 59, 255-278.

Feeney, A., \& Heit, E. (2007). Inductive reasoning: Experimental, developmental, and computational approaches. Cambridge, NY: Cambridge University Press.

Feuerstein, R., Feuerstein, R. S., Falik, L. H., \& Rand, Y. (2002). The dynamic assessment of cognitive modifiability: The learning propensity assessment device, theory, instruments, and techniques. Jerusalem, Israel: International Centre for the Enhancement of Learning Potential Press.

Feuerstein, R., Rand, Y., Hoffman, M. B., \& Miller, R. (1980). Instrumental enrichment: An intervention program for cognitive modifiability. Baltimore, MD: University Park Press.

Galotti, K. M., Komatsu, L. K., \& Voelz, S. (1997). Children's differential performance on deductive and inductive syllogisms. Developmental Psychology, 33, 70-78.

Gentner, D. (1988). Metaphor as structure mapping: The relational shift. Child Development, 59, 47-59.

Gentner, D., \& Holyoak, K. J. (1997). Reasoning and learning by analogy. The American Psychologist, 52, 32-34.

Goel, V., \& Dolan, R. J. (2004). Differential involvement of left prefrontal cortex in inductive and deductive reasoning. Cognition, 93, B109-B121.

Goodman, N. (1955). Fact, fiction, and forecast. Cambridge, MA: Harvard University Press.

Gopnik, A., \& Wellman, H. (1994). The theory-theory. In L. Hirschfeld \& S. Gelman (Eds.), Mapping the mind: Domain specificity in cognition and culture (pp. 257-293). New York, NY: Cambridge University Press.

Goswami, U. (Ed.). (2010). Inductive and deductive reasoning. In The Wiley-Blackwell handbook of childhood cognitive development (pp. 399-419). London, United Kingdom: Wiley.

Halford, G. S., \& Andrews, G. (2006). Reasoning and problem solving. In W. Damon, R. Lerner, D. Kuhn, \& R. S. Siegler (Eds.), Handbook of child psychology: Vol. 2. Cognition, perception, and language (6th ed., pp. 557-608). Hoboken, NJ: Wiley. 
Halford, G. S., Baker, R., McCredden, J. E., \& Bain, J. D. (2005). How many variables can humans process? Psychological Science, 16, 70-76.

Halford, G. S., Wilson, W. H., \& Phillips, S. (1998). Processing capacity defined by relational complexity: Implications for comparative, developmental, and cognitive psychology. The Behavioral and Brain Sciences, 21, 803-865.

Halford, G. S., Wilson, W. H., \& Phillips, S. (2010). Relational knowledge: The foundation of higher cognition. Trends in Cognitive Sciences, 14, 497-505.

Heit, E. (2007). What is induction and why study it? In A. Feeney \& E. Heit (Eds.), Inductive reasoning: Experimental, developmental, and computational approaches (pp. 1-24). New York, NY: Cambridge University Press.

Heit, E., \& Rotello, C. M. (2010). Relations between inductive reasoning and deductive reasoning. Journal of Experimental Psychology: Learning, Memory, and Cognition, 36, 805-812.

Hessels-Schlatter, C. (2010). Development of a theoretical framework and practical application of games in fostering cognitive and metacognitive skills. Journal of Cognitive Education and Psychology, 9, 116-138.

Hofstadter, D. R. (2001). Epilogue: Analogy as the core of cognition. In D. Gentner, K. J. Holyoak, \& B. N. Kokinov (Eds.), The analogical mind: Perspectives from cognitive science (pp. 499-538). Cambridge, MA: Massachusetts Institute of Technology Press.

Holland, J. H., Holyoak, K. J., Nisbett, R. E., \& Thagard, P. (1986). Induction: Processes of inference, learning, and discovery. Cambridge, MA: Massachusetts Institute of Technology Press.

Holyoak, K. J., \& Thagard, P. (1997). The analogical mind. The American Psychologist, 52, 35-44.

Houdé, O. (2000). Inhibition and cognitive development: Object, number, categorization, and reasoning. Cognitive Development, 15, 63-73.

Jarosz, A. F., \& Wiley, J. (2012). Why does working memory capacity predict RAPM performance? A possible role of distraction. Intelligence, 40, 427-438.

Kagan, J., Pearson, L., \& Welch, L. (1966). Conceptual impulsivity and inductive reasoning. Child Development, 584-594.

Karpov, Y. V. (2013). A way to implement the neo-Vygotskian theoretical learning approach in the schools. International Journal of Pedagogical Innovations, 1, 25-35.

Klauer, K. J., \& Phye, G. D. (1994). Cognitive training for children: A developmental program of inductive reasoning and problem solving. Seattle, WA: Hogrefe.

Klauer, K. J., \& Phye, G. D. (2008). Inductive reasoning. A training approach. Review of Educational Research, 78, 85-123.

Klauer, K. J., Willmes, K., \& Phye, G. D. (2002). Inducing inductive reasoning: Does it transfer to fluid intelligence? Contemporary Educational Psychology, 27, 1-25.

Kokis, J. V., Macpherson, R., Toplak, M. E., West, R. F., \& Stanovich, K. E. (2002). Heuristic and analytic processing: Age trends and associations with cognitive ability and cognitive styles. Journal of Experimental Child Psychology, 83, 26-52.

Kuhn, D. (2013). Reasoning. In P. D. Zelazo (Ed.), The Oxford handbook of developmental psychology (pp. 744-760). Oxford, NY: Oxford University Press.

Kuhn, D., Katz, J. B., \& Dean, D. (2004). Developing reason. Thinking and Reasoning, 10, 197-219.

Lawson, C. A., \& Fisher, A. V. (2009). When sample size matters: The influence of sample size on category variability on children's and adults inductive reasoning. In N. Taatgen \& H. van Rijn (Eds.), Proceedings of the 31st Annual Meeting of the Cognitive Science Society (pp. 1324-1329). Amsterdam, The Netherlands: Cognitive Science Society.

Lawson, C. A., \& Fisher, A. V. (2011). It's in the sample: The effects of sample size on the development of inductive generalization. Journal of Experimental Child Psychology, 110, 499-519.

Leech, R., Mareschal, D., \& Cooper, R. P. (2007). Relations as transformations: Implications for analogical reasoning. Quarterly Journal of Experimental Psychology, 60, 897-908. 
Mackey, A. P., Hill, S. S., Stone, S. I., \& Bunge, S. A. (2011). Differential effects of reasoning and speed training in children. Developmental Science, 14, 582-590.

Markman, A. B., \& Gentner, D. (2001). Thinking. Annual Review of Psychology, 52, 223-247.

Molnár, G. (2011). Playful fostering of 6- to 8-year-old students' inductive reasoning. Thinking Skills and Creativity, 6, 91-99.

Molnár, G., Greiff, S., \& Csapó, B. (2013). Inductive reasoning, domain specific and complex problem solving: Relations and development. Thinking Skills and Creativity, 9, 35-45.

Morrison, R. G., Doumas, L. A., \& Richland, L. E. (2011). A computational account of children's analogical reasoning: Balancing inhibitory control in working memory and relational representation. Developmental Science, 14, 516-529.

Moshman, D. (1995). Reasoning as self-constrained thinking. Human Development, 38, 53-64.

Moshman, D. (2004). From inference to reasoning: The construction of rationality. Thinking and Reasoning, 10, 221-239.

Nisbett, R. E. (2009). Intelligence and how to get it. New York, NY: Norton.

Nisbett, R. E., Fong, G. T., Lehman, D. R., \& Cheng, P. W. (1987). Teaching reasoning. Science, 238, $625-631$.

Oakland, T., \& Hu, S. (1992). The top ten tests used with children and youth worldwide. Bulletin of the International Test Commission, 19, 99-120.

Oberauer, K., Süb, H.-M., Wilhelm, O., \& Wittmann, W. W. (2008). Which working memory function predicts intelligence? Intelligence, 36, 641-652.

Paour, J.-L. (1992). Induction of logic structures in the mentally retarded: An assessment and intervention instrument. In H. C. Haywood \& D. Tzuriel (Eds.), Interactive assessment (pp. 119-166). New York, NY: Springer Publishing.

Paour, J.-L. (1995). Entraîner les fondements du raisonnement inductif et analogique [Cognitive training of inductive and analogical reasoning]. In F. Büchel (Ed.), Léducation cognitive [Cognitive education] (pp. 267-283). Lausanne, Switzerland: Delachaux et Niestlé.

Perkins, D. (1995). Outsmarting IQ: The emerging science of learnable intelligence. New York, NY: Free Press.

Perkins, D., Tishman, S., Ritchart, R., Donis, K., \& Andrade, A. (2000). Intelligence in the wild: A dispositional view of intellectual traits. Educational Psychology Review, 12, 269-293.

Perret, P., Dauvier, B., Bailleux, C., \& Thomachot, L. (2013). Intégration relationnelle en mémoire de travail et intelligence fluide chez l'enfant [Relational integration in working memory and children's fluid intelligence]. L’Année Psychologique/Topics in Cognitive Psychology, 113, 191-212.

Phye, G. D. (1990). Inductive problem solving: Schema inducement and memory-based transfer. Journal of Educational Psychology, 82, 826-831.

Pillow, B. H., \& Pearson, R. M. (2012). Children's evaluation of the certainty of another person's inductive inferences and guesses. Cognitive Development, 27, 299-313.

Rattermann, M. J., \& Gentner, D. (1998). More evidence for a relational shift in the development of analogy: Children's performance on a causal-mapping task. Cognitive Development, 13, 453-478.

Raven, J., Raven, J. C., \& Court, J. H. (1998). Manual for Raven's Progressive Matrices and Vocabulary Scales. Section 1: General overview. San Antonio, TX: Harcourt Assessment.

Rothbart, M. K., \& Rueda, M. R. (2005). The development of effortful control. In U. Mayr, E. Awh, \& S. W. Keele (Eds.), Developing individuality in the human brain: A tribute to Michael I. Posner (pp. 167-188). Washington, DC: American Psychological Association.

Rueda, M. R., Rothbart, M. K., Saccamanno, L., \& Posner, M. I. (2005). Training, maturation and genetic influences on the development of executive attention. Proceedings of the National Academy of Sciences, USA, 102, 14931-14936.

Schulz, L. E., Goodman, N. D., Tenenbaum, J. B., \& Jenkins, C. A. (2008). Going beyond the evidence: Abstract laws and preschoolers' responses to anomalous data. Cognition, 109, 211-223. 
Shipstead, Z., Hicks, K. L., \& Engle, R. W. (2012). Cogmed working memory training: Does the evidence support the claims? Journal of Applied Research in Memory and Cognition, 1, 185-193.

Siegler, R. S. (1996). Emerging minds: The process of change in children's thinking. New York, NY: Oxford University Press.

Siegler, R. S. (2000). The rebirth of children's learning. Child Development, 71, 26-35.

Siegler, R. S., \& Stern, E. (1998). A microgenetic analysis of conscious and unconscious strategy discoveries. Journal of Experimental Psychology: General, 127, 377-397.

Snow, R. E. (1980). Aptitude processes. In R. E. Snow, P.-A. Federico, \& W. E. Montague (Eds.), Aptitude, learning, and instruction: Cognitive process analyses of aptitude (pp. 27-63). Hillsdale, NJ: Lawrence Erlbaum Associates.

Spearman, C. (1927). The nature of "intelligence" and the principles of cognition. London, United Kingdom: MacMillan.

Sternberg, R. J. (1983). Natural, unnatural, and supernatural concepts. Cognitive Psychology, 14, 451-488.

Sternberg, R. J. (1986). Toward a unified theory of human reasoning. Intelligence, 10, 281-314.

Sternberg, R. J., \& Gardner, M. K. (1983). Unities in inductive reasoning. Journal of Experimental Psychology: General, 112, 80-116.

Stroop, J. R. (1935). Studies of interference in serial verbal reactions. Journal of Experimental Psychology, $18,643-661$.

Thibaut, J.-P., French, R., \& Vezneva, M. (2010). The development of analogy making in children: Cognitive load and executive functions. Journal of Experimental Child Psychology, 106, 1-19.

Thomas, M. S. C., \& Mareschal, D. (2001). Metaphor as categorisation: A connectionist implementation. Metaphor and Symbol, 16, 5-27.

Thorell, L. B., Lindqvist, S., Bergman Nutley, S., Bohlin, G., \& Klingberg, T. (2009). Training and transfer effects of executive functions in preschool children. Developmental Science, 12, 106-113.

Tomic, W., \& Kingma, J. (1998). Accelerating intelligence development through inductive reasoning training. Advances in Cognition and Educational Practice, 5, 291-305.

Verguts, T., \& De Boeck, P. (2002). The induction of solution rules in Raven's Progressive Matrices Test. European Journal of Cognitive Psychology, 14, 521-547.

Vigneau, F., Caissie, A., \& Bors, D. A. (2006). Eye-movement analysis demonstrates strategic influences on individual differences in intelligence. Intelligence, 34, 261-272.

Viskontas, I. V., Morrison, R. G., Holyoak, K. J., Hummel, J. E., \& Knowlton, B. J. (2004). Relational integration, inhibition, and analogical reasoning in older adults. Psychology and Aging, 19, 581-591.

Vodegel-Matzen, L., van der Molen, M., \& Dudink, A. (1994). Error analysis of Raven test performance. Personality and Individual Differences, 16, 433-445.

Whitebread, D. (1996). The development of children's strategies on an inductive reasoning task. British Journal of Educational Psychology, 66, 1-21.

Wiley, J., Jarosz, A. F., Cushen, P. J., \& Colflesh, G. J. H. (2011). New rule use drives the relation between working memory capacity and Raven's Advanced Progressive Matrices. Journal of Experimental Psychology: Learning, Memory, and Cognition, 37, 256-263.

Yeager, D. S., \& Dweck, C. S. (2012). Mindsets that promote resilience: When students believe that personal characteristics can be developed. Educational Psychologist, 47, 302-314.

Zelazo, P. D. (Ed.). (2013). Developmental psychology: A new synthesis. In The Oxford handbook of developmental psychology (pp. 3-34). Oxford, NY: Oxford University Press.

Correspondence regarding this article should be directed to Patrick Perret, Aix Marseille Université, Maison de la Recherche, Faculté des Arts, Lettres, Langues et Sciences Humaines, 29, av. Robert Schuman 13 621- Aix-en-Provence Cedex 1, France. E-mail: Patrick.Perret@univ-amu.fr 